Published in final edited form as:

Conf Proc IEEE Eng Med Biol Soc. 2008 ; 2008: 965-968. doi:10.1109/IEMBS.2008.4649315.

\title{
In-Vitro Evaluation of Sensors and Amplifiers to Measure Left Ventricular Pressure in Mice
}

\author{
Craig J. Hartley, Anilkumar K. Reddy, and George E. Taffet \\ Sections of Cardiovascular Sciences \& Geriatrics, Dept. of Medicine, Baylor College of Medicine; \\ and The DeBakey Heart and Vascular Center of The Methodist Hospital, Houston, TX 77030
}

\section{Abstract}

Mice are becoming more common as research models, and several companies now manufacture sensors and instrumentation to measure left ventricular (LV) pressure and volume in mice. It is often assumed that pressure is easier to measure than volume, and that all sensors perform similarly, but there are differences. We measured in-vitro the frequency and step responses, immersion response, stability, accuracy, linearity, and sensitivity to lateral or bending force of several solid-state sensors and amplifiers commonly used in mice. We tested 4 micro-sensors each from Millar, Scisense, and RADI, and also fluid-filled catheters. All solid-state sensors were stable with drifts of $<1 \mathrm{mmHg} / \mathrm{hr}$, had flat frequency response to $>1 \mathrm{kHz}$, and were accurate and linear to within $+/-2 \mathrm{mmHg}$ from $0-300 \mathrm{mmHg}$. The frequency response of the fluid-filled catheter was down by $50 \%$ at $30 \mathrm{~Hz}$. The amplifiers from Millar, Scisense, and RADI, had time delays of 0.2, 3.2 and $10.6 \mathrm{~ms}$ respectively. The Millar and RADI sensors were unresponsive to lateral forces, but the Scisense catheters had sensitivities as high as $5.3 \mathrm{mmHg} / \mathrm{g}$. There are significant differences in solid state pressure sensors and amplifiers which could generate offsets, time delays, and distortions which could go unrecognized in-vivo.

\section{Keywords}

blood pressure; frequency response; fidelity

\section{Introduction}

It is now possible in mice to simuate human diseases and conditions affecting the heart and vascular systems, but it has been difficult in the past to make reliable measurements of pressure, flow, dimensions, and volume. Cardiac function is often assessed by calculating indices based on left ventricular (LV) pressure $(\mathrm{P})$ and volume $(\mathrm{V})$ measured throughout the cardiac cycle [1]. Several companies now market P-V catheters for applications in mice [2]. It is generally accepted that LV volume via conductance is more difficult to measure than LV pressure [3] because solid-state pressure sensors have been around for nearly 40 years [4]. However, measuring LV pressure in small ventricles with solid-state sensors is not simple, and the requirements for accuracy and stability have not been established for these miniature sensors. Therefore, the goal of this study was to perform in-vitro and in-vivo testing of 1.0-1.5 French pressure and P-V solid state sensors and amplifiers purchased from Millar Instruments, Scisense, and RADI Medical Systems to assess stability, linearity, sensitivity, accuracy, and fidelity.

\section{Methods}

Figure 1 shows photos of the sensors evaluated. Two samples of each type of sensor were tested. The Millar and Scisense pressure and P-V sensors are intended for applications in mice. The RADI sensors are mounted on guide wires designed for applications in human 
coronary arteries but can be used in mice with modifications [5]. A Meritrans sensor (not shown) is an external sensor used with fluid-filled catheters. Each sensor contains 2 resistors bonded to a diaphragm or beam which deforms and stresses one or both resistors when pressure is applied, and the change in resistance is a function of pressure. The resistors are wired as part of a Wheatstone bridge, and the other two bridge resistors (and several other fixed and variable resistors) are located in the electrical connector. The variable resistors are set during manufacture to minimize sensitivity to temperature, to balance the bridge at zero pressure, and to set the sensitivity to a specific value.

Figure 2 shows a simplified schematic of a pressure amplifier and photos of the Millar and Scisense compensation networks. We tested amplifiers from each manufacturer and also used one of our own (BCM) designed with minimal filtration. The BCM amplifier has potentiometers for balance and gain, a separate one for sensor balance, a switch to replace the sensor with a reference bridge, and a switch to offset the reference bridge by $100 \mathrm{mmHg}$. The sensors all look like 4-element "diamond" bridges to the amplifier, and the cross-type reference bridge is designed to match the impedance of the sensor bridge while being inherently balanced. When the reference bridge is connected to the amplifier, the amplifier can be balanced to output zero volts at zero pressure and then the gain can be set to output 1 volt at $100 \mathrm{mmHg}$. When the sensor is selected and is at zero pressure, the sensor balance can be adjusted to match the reference zero. By this means sensor drift can be isolated from amplifier drift. The Millar and Scisense amplifiers have fixed amplifier balance and gain, but are otherwise similar.

We measured the frequency response from 0 to $1 \mathrm{kHz}$ and the step response of each amplifier using an electronic signal generator connected to the sensor input. We tested each sensor using a mechanical actuator to pressurize a small chamber into which each sensor was inserted. We tested the linearity and gain of each sensor in air from 0 to $300 \mathrm{mmHg}$ in $10 \mathrm{mmHg}$ steps using a mercury manometer and a cuff inflation bulb. To test long-term stability and sensitivity to immersion, we first powered each gauge on for $30 \mathrm{~min}$, balanced in air, recorded for $30 \mathrm{~min}$, and then immersed in water for 5 hours.

Some solid-state gauges are sensitive to lateral or bending forces applied to the housing away from the diaphragm. To quantify this effect we inserted each gauge through a small stiff tube with just the sensor and housing extending from the tube, pushed the gauge head against a laboratory scale, and measured the "pressure" reading in $\mathrm{mmHg}$ versus the scale reading in grams with the sensor oriented for maximum positive and maximum negative readings. Figure 3 shows a photo of the scale with a sensor pushing against the platform.

We also tested Millar and Scisense PV catheters in 4 mice to see if the sensitivity to side forces could distort LV pressure readings in-vivo. We inserted each gauge into a mouse LV via the right carotid artery and then rotated the catheter through $360^{\circ}$ in both directions while recording $\mathrm{LV}$ pressure and $\mathrm{dP} / \mathrm{dt}$ using the BCM amplifier.

\section{Results}

\section{Amplifiers}

Figure 4 shows the responses of the 4 amplifiers to a voltage step applied to each input.

Shown on the figure are the times to reach half amplitude which range from 0.05 to $10.6 \mathrm{~ms}$. Figure 5 shows the responses to a sine wave swept from $1 \mathrm{~Hz}$ to $1 \mathrm{kHz}$. The fine-scale modulation is due to under-sampling of the signals by the oscilloscope. The half amplitude (-6 dB point) is $6 \mathrm{kHz}$ for BCM, $1.2 \mathrm{kHz}$ for Millar, $300 \mathrm{~Hz}$ for Scisense, and $600 \mathrm{~Hz}$ for RADI. The BCM reference amplifier had the shortest delay and highest frequency response, 
the RADI amplifier had the longest time delay, and the Scisense amplifier had the lowest frequency response.

\section{Sensors}

The frequency response of each sensor was tested using an air-coupled pressure generator attached to a small Luer fitting which could hold two sensors. The sensors were inserted into mating Luer fittings with O-ring seals which were then attached to the generator. The pressure generator was driven with a sine wave swept from $1 \mathrm{~Hz}$ to $1 \mathrm{kHz}$, and an older 3-F Millar gauge was used to verify the fidelity of the pressure generator. The BCM amplifier was used for this test. Figure 6 shows the responses of all catheter-tip sensors plus the Meritrans external gauge connected directly and through a PE50 tube as it might be connected to a mouse. Except for the Meritrans, all sensors show flat responses to at least 1 $\mathrm{kHz}$

\section{Immersion and stability}

Figure 7 shows the responses to immersion in water for each sensor for 6 hours after $30 \mathrm{~min}$ of stabilization in air. To minimize hydrostatic effects, each sensor was placed in the O-ring sealed Luer fitting, open to air, and water was added with a syringe to wet the sensor. Two of the Millar sensors showed large offsets upon wetting, but were stable after $30 \mathrm{~min}$ of immersion. Both sensors had been previously used in mice, were subsequently cleaned more carefully, and the offset upon immersion disappeared. After $30 \mathrm{~min}$ of stabilization following immersion (per instructions from each manufacturer), all sensors were stable for over 5 hours with drifts of less than $1 \mathrm{mmH}$ g/hour.

\section{Gain and Linearity}

Each sensor was linear to within $2 \mathrm{mmHg}$ from 0 to $300 \mathrm{mmHg}$ against a mercury manometer and met the industry standard sensitivity of $5 \mu \mathrm{V} / \mathrm{V}) / \mathrm{mmH} \mathrm{g}$.

\section{Lateral force}

All of the Scisense catheters were sensitive to lateral forces applied to the tip of the catheter but away from the pressure sensing membrane. Figure 8 shows a plot of the "pressure reading" versus the lateral force for a Scisense and a Millar PV catheter using the apparatus shown in Figure 3. The lateral sensitivity of the Scisense catheter is $\sim 5.3 \mathrm{mmHg} / \mathrm{g}$, while that of the Millar is $0.75 \mathrm{mmH} \mathrm{g/g}$.

Figure 9 shows examples of LV pressure signals from a mouse using both Millar and Scisense P-V sensors showing general agreement between the signals. In one mouse peak LV pressure varied from 82-117 $\mathrm{mmHg}$ with rotation of the Scisense, but not the Millar catheter. In the other mice, smaller variations were found with both catheters.

\section{Discussion}

The magnitude of high frequency components in the pressure signal is highest at the heart and decreases with distance from the heart. When derivatives are calculated, the magnitude of each harmonic is multiplied by its relative frequency. It is generally accepted that 10-20 harmonics are required to reproduce the waveform of an aortic pressure signal, but that more (perhaps 25-50) are required to accurately reproduce LVP and to calculate its derivative (dP/dt) [6]. Although the slope of LVP in Figure 9 is modest at $\sim 6 \mathrm{mmHg} / \mathrm{ms}(6,000 \mathrm{mmHg} /$ $\mathrm{s}), \mathrm{dP} / \mathrm{dt}$ can exceed $25 \mathrm{mmHg} / \mathrm{ms}$ in mice at high heart rates with inotropic stimulation. 


\section{Amplifiers}

The step and frequency responses of the Millar amplifier were nearly equal to those of the BCM reference, but the amplifiers from RADI and Scisense had longer time delays and rise times and lower frequency responses. It is not clear that the limited frequency response (passing 25-50 harmonics) is a significant limitation, but the time delays of 3.2 and $10.6 \mathrm{~ms}$ are significant in mice if timing with respect to other signals is important. In addition, the 30 $\mathrm{mmHg} / \mathrm{ms}$ rise-time of the Scisense amplifier is only slightly above peak $\mathrm{dP} / \mathrm{dt}$ under inotropic stimulation. The reason given by Scisense for the limited amplifier response is to match the response of the volume amplifier such that pressure-volume loops are not distorted.

\section{Sensors}

The frequency response of all of the solid-state sensors was flat to over $1 \mathrm{kHz}$, while that of the external gauge connected via catheter was severely limited. It should also be noted that the sensitivity to hydrostatic pressure of solid-state sensors is not a serious concern in a mouse where the size of the heart is so small.

\section{Immersion and stability}

For maximum stability and to minimize the offset upon immersion, each sensor should be carefully cleaned after use to remove any debris which could solidify on the surface of the diaphragm. Stabilization and balancing to zero should also be done for 30 min with the sensor immersed in saline, preferably at body temperature.

\section{Gain and linearity}

The gain and linearity of all gauges tested met the industry standard of $5(\mu \mathrm{V} / \mathrm{V}) / \mathrm{mmH}$. However, the Scisense gauges were special ordered for use with a Millar amplifier, and standard Scisense gauges are set to a different sensitivity. Indeed, our gauges, when used with a Scisense amplifier, were off by $\sim 20 \%$. Thus, care must be taken when using a gauge calibrated for a different amplifier. We normally adjust our amplifiers to read 0 volts at zero pressure and 1.0 volts at $100 \mathrm{mmHg}$ to make calibration of the recording or data acquisition system simpler. This can be done with Millar, but not with Scisense amplifiers which have an output of -2.86 volts at 0 and -0.57 volts at $100 \mathrm{mmHg}$.

\section{Lateral force}

A surprising finding was the relatively high sensitivity of the Scisense catheters to the magnitude and direction of lateral forces applied to the tip of the catheter. Given that ventricular pressure ranges from 0 to about $100 \mathrm{mmHg}$ or $1.4 \mathrm{~g} / \mathrm{mm}^{2}$ during a cardiac cycle, the dynamic forces pushing against the catheter tip may generate synchronous distortions to the $\mathrm{LV}$ pressure signal recorded from a mouse. Because all of the sensors tested are essentially rigid with respect to the arterial system of a mouse, inserting a catheter into the $\mathrm{LV}$ via the right carotid artery forces the normally curved path into a nearly straight line. The lateral force required to do this in a beating heart may be on the order of several grams but will be highly dependent on the specific anatomy of a given mouse. We were unable to measure the magnitude of the distortion in-vivo, but did notice subtle differences in the shape of peak LV pressure measured by Millar and Scisense catheters as shown in Figure 9.

\section{Conclusions}

It is important to recognize and understand the limitations of any measurement system, and we have attempted to quantify the characteristics of sensors to measure LV pressure in mice. These catheter-tip sensors are large and stiff relative to the mouse LV cavity, and the 
resulting distortions of the anatomy during insertion and to the contraction of the heart could be significant. All of the sensors tested appear to have the necessary accuracy, linearity, and stability to measure LV pressure in mice for several hours, and the sensors themselves all have excellent frequency response. However, some of the amplifiers can introduce delays and limitations to frequency response which can be significant when accurate timing and the calculation of derivatives are important. The lateral force sensitivity of Scisense catheters is particularly troubling because any resulting distortion of the LV pressure signal will be synchronous with the cardiac cycle and difficult to detect.

\section{Acknowledgments}

The authors wish to thank Thuy Pham, James A. Brooks, and Ross J. Hartley for their valuable contributions to this research and Millar Instruments, RADI Medical Systems, and Scisense for supplying catheters and amplifiers. This work was supported in part by National Institutes of Health Grants HL22512 (Hartley), AG 17899 (Taffet), HL73041 (Reddy).

\section{References}

1. Suga H, Sagawa K, Shoukas AA. Load independence of the instantaneous pressure volume ratio of the canine left ventricle and effects of epinephrine on the ratio. Circulation Research. 1973; 32:314322. [PubMed: 4691336]

2. Georgakopoulos D, Mitzner WA, Chen CH, Byrne BJ, Millar HD, Hare JM, Kass DA. In vivo murine left ventricular pressure-volume relations by miniaturized conductance micromanometry. Am J Physiol Heart Circ Physiol. 1998; 274:H1414-H1422.

3. Burkhoff D, van der Velde ET, Kass D, Baan J, Maughan WL, Sagawa K. Accuracy of volume measurement by conductance catheter in isolated, ejecting canine hearts. Circulation. 1985; 72(2): 440-447. [PubMed: 4006150]

4. Millar HD, Baker LE. A stable ultraminiature catheter-tip pressure transducer. Med Biol Engng. 1973; 11:86-89. [PubMed: 4690340]

5. Reddy AK, Li Y-H, Pham TT, Ochoa LN, Trevino MT, Hartley CJ, Michael LH, Entman ML, Taffet GE. Measurement of aortic input impedance in mice: effects of age on aortic stiffness. Am J Physiol Heart Circ Physiol. 2003; 285:H1464-H1470. [PubMed: 12775560]

6. Nichols, WW.; O’Rourke, MF. McDonald's Blood Flow in Arteries: Theoretical, Experimental, and Clinical Principles. 4. London: Edward Arnold; 1998. p. 201-222. 


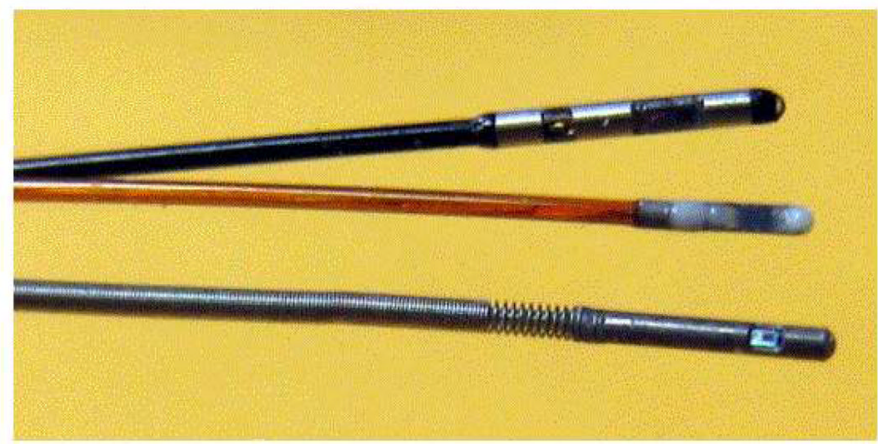

Pressure

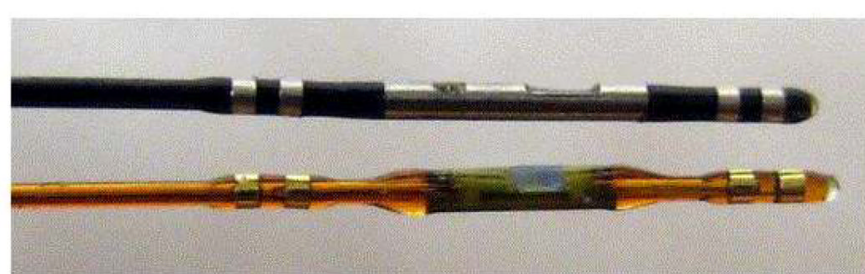

Press/Nol

Scisense

$1.2 \mathrm{~F}$

Millar

1.4F

Figure 1.

Photos of the pressure and pressure/volume sensors tested. 


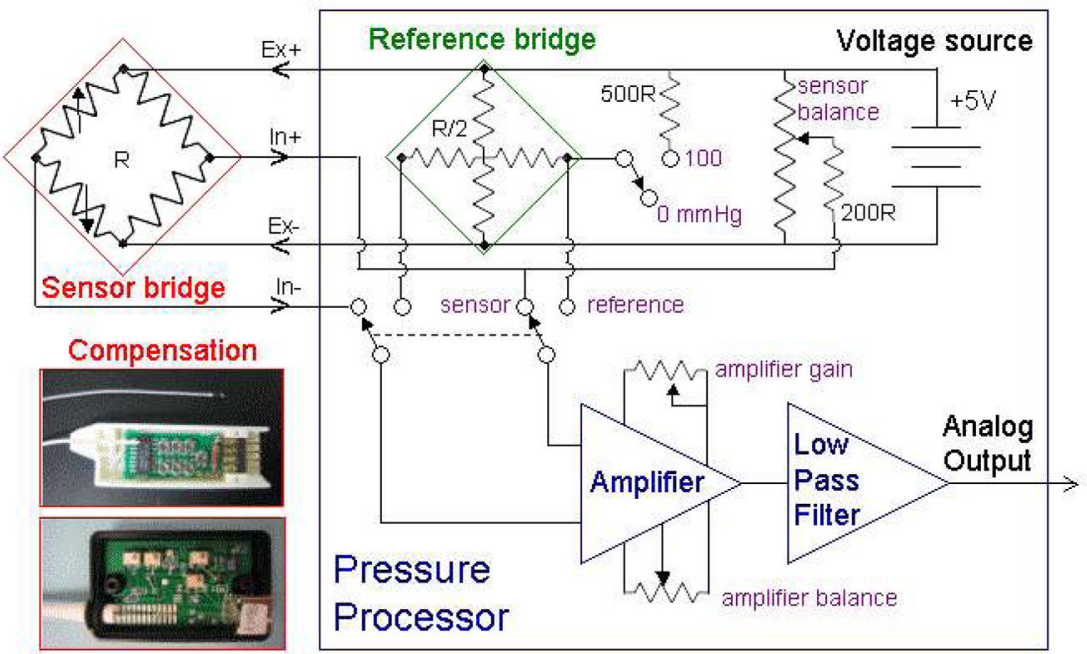

Figure 2.

Simplified block diagram of a pressure amplifier. 

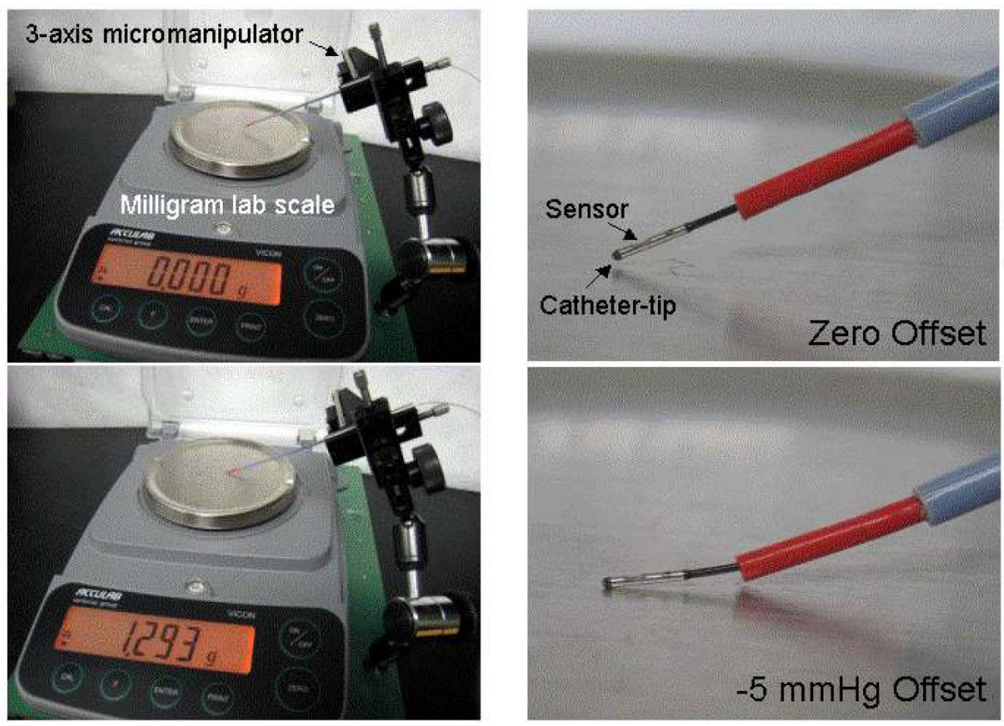

Figure 3.

Set-up to measure sensitivity to lateral forces applied to the sensor tip. We measured the offset in $\mathrm{mmHg}$ versus the force on the tip in grams. 

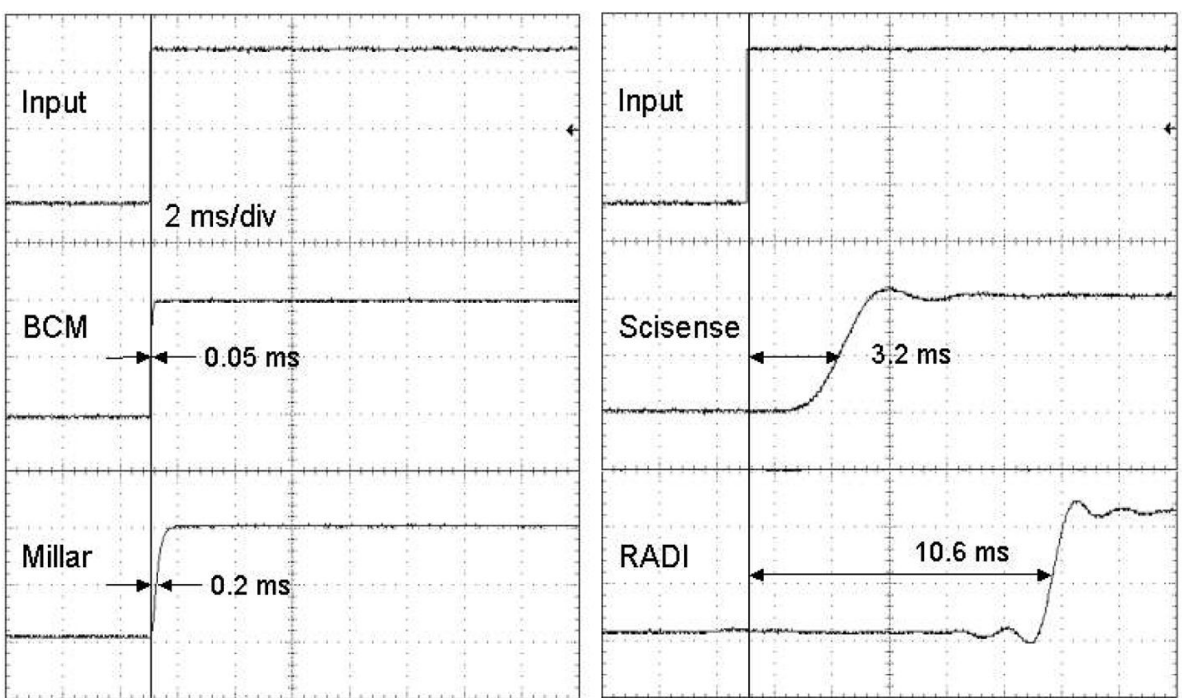

Figure 4.

Responses of the four amplifiers to the input step shown above. The delay time to reach $1 / 2$ amplitude is shown for each amplifier. 


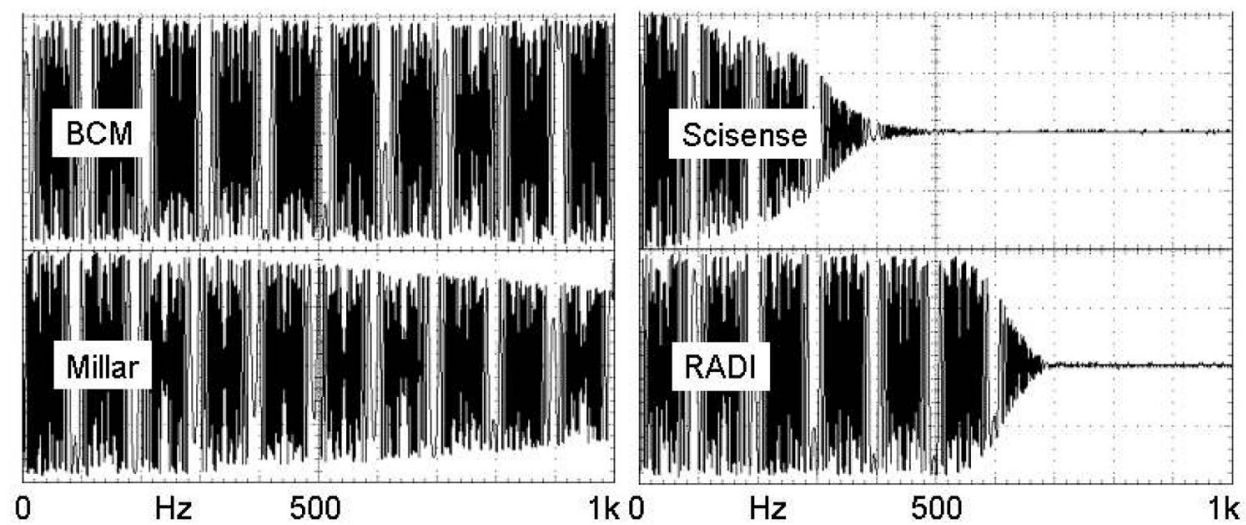

Figure 5.

Frequency response from $1 \mathrm{~Hz}$ to $1 \mathrm{kHz}$ for each amplifier. The modulation is due to undersampling by the storage oscilloscope. 


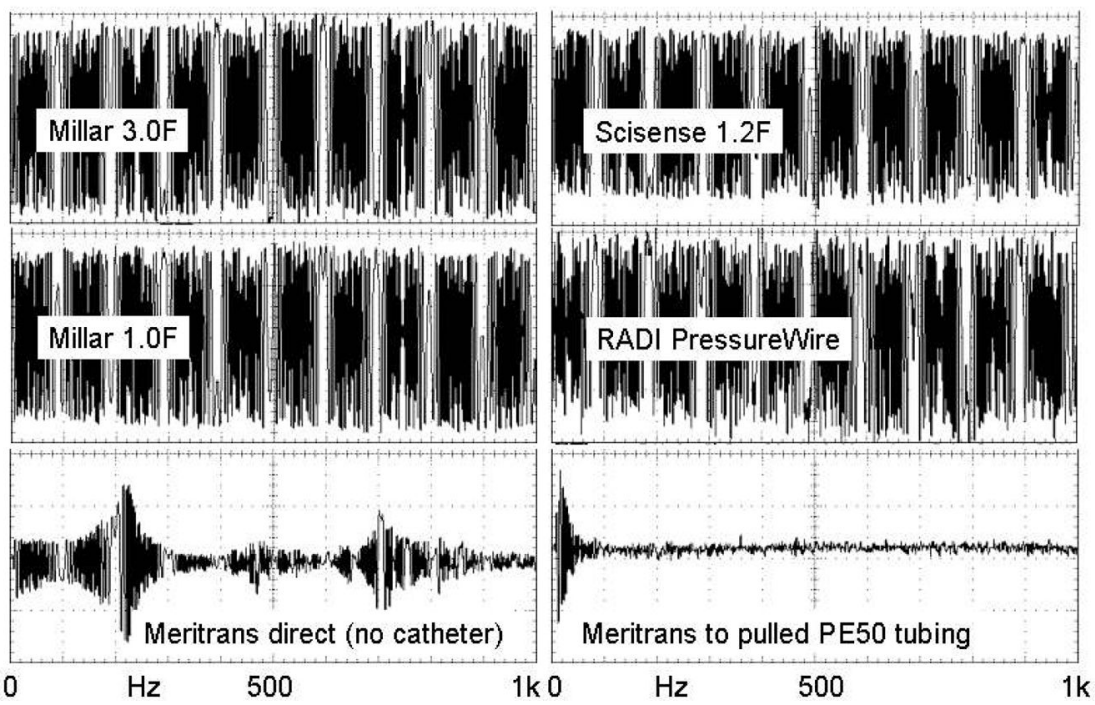

Figure 6.

Frequency response of each sensor connected to the BCM amplifier. 


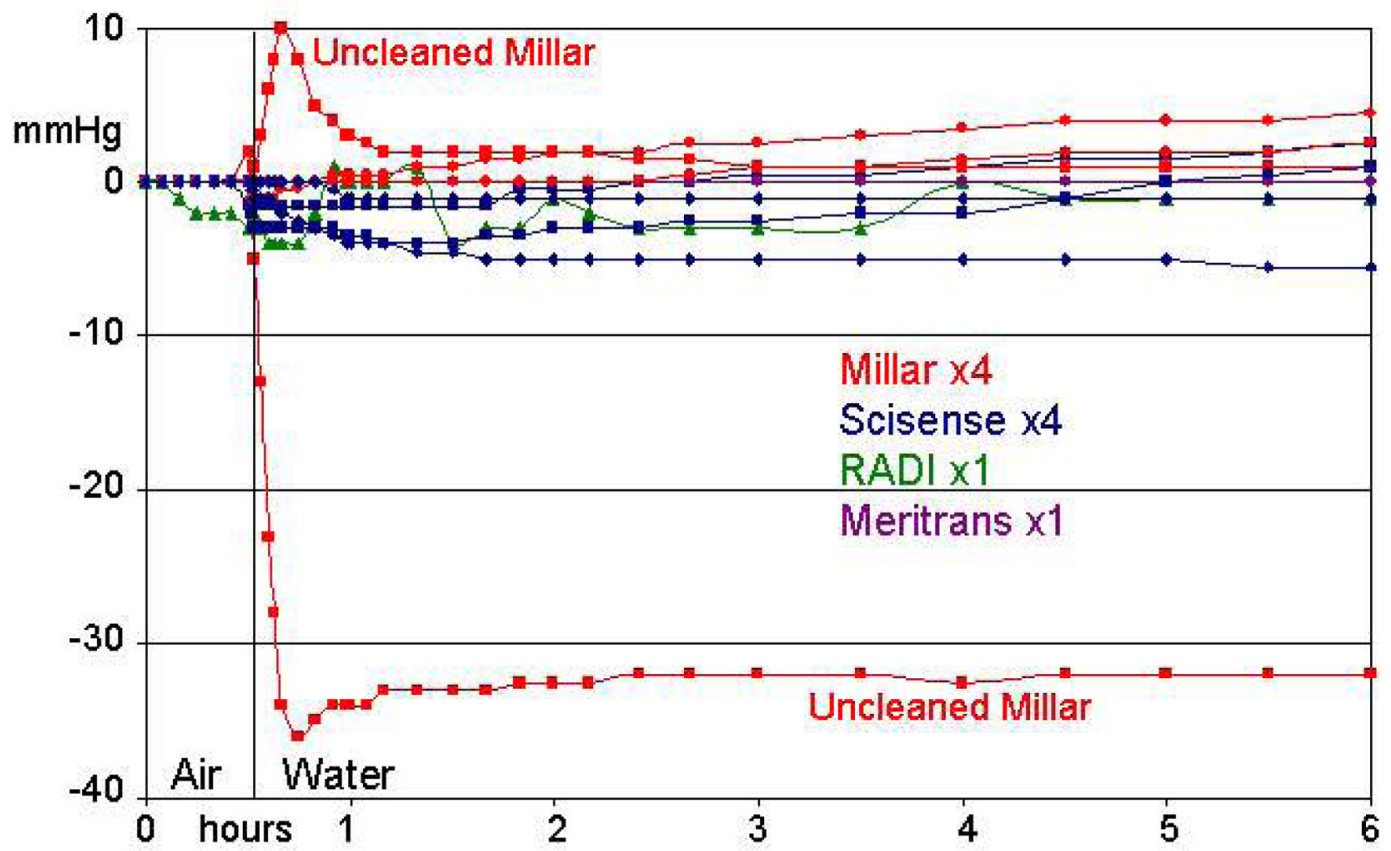

Figure 7.

Response of each sensor after stabilizing in air to immersion in water for 6 hours. 


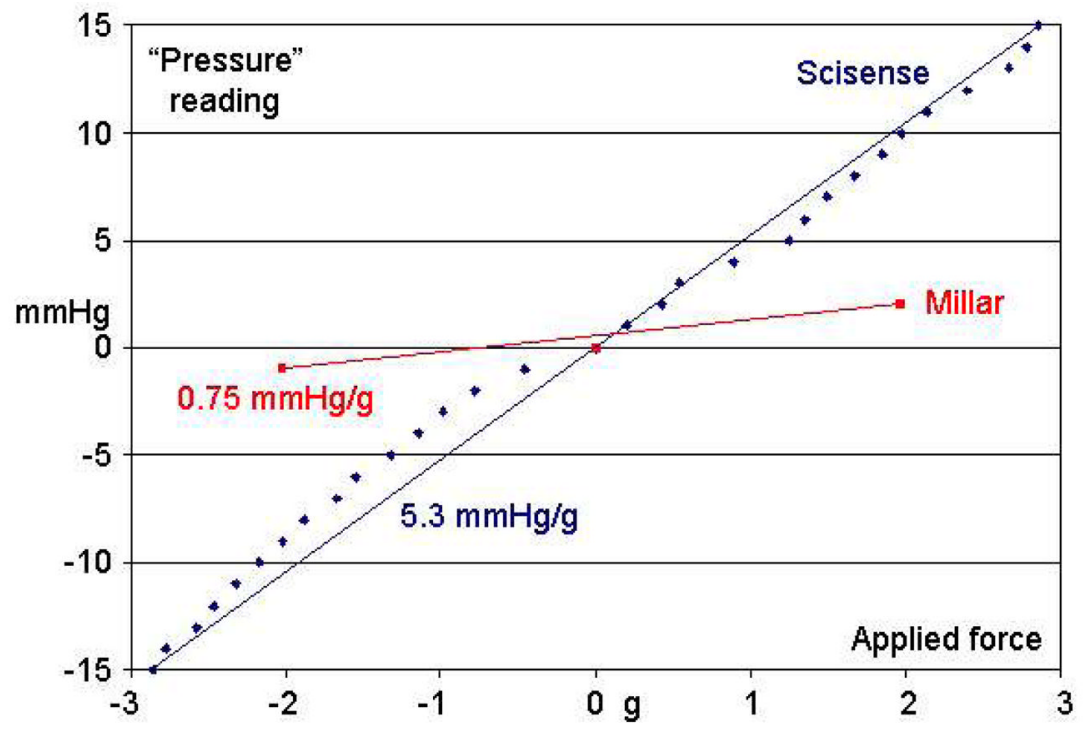

Figure 8.

Offset in mmHg versus applied lateral force to the catheter tip in grams for a Scisense and a Millar pressure/volume catheter. Two runs were made for each catheter in opposite directions with the catheter oriented to generate the maximum positive or negative pressure reading versus applied force. 

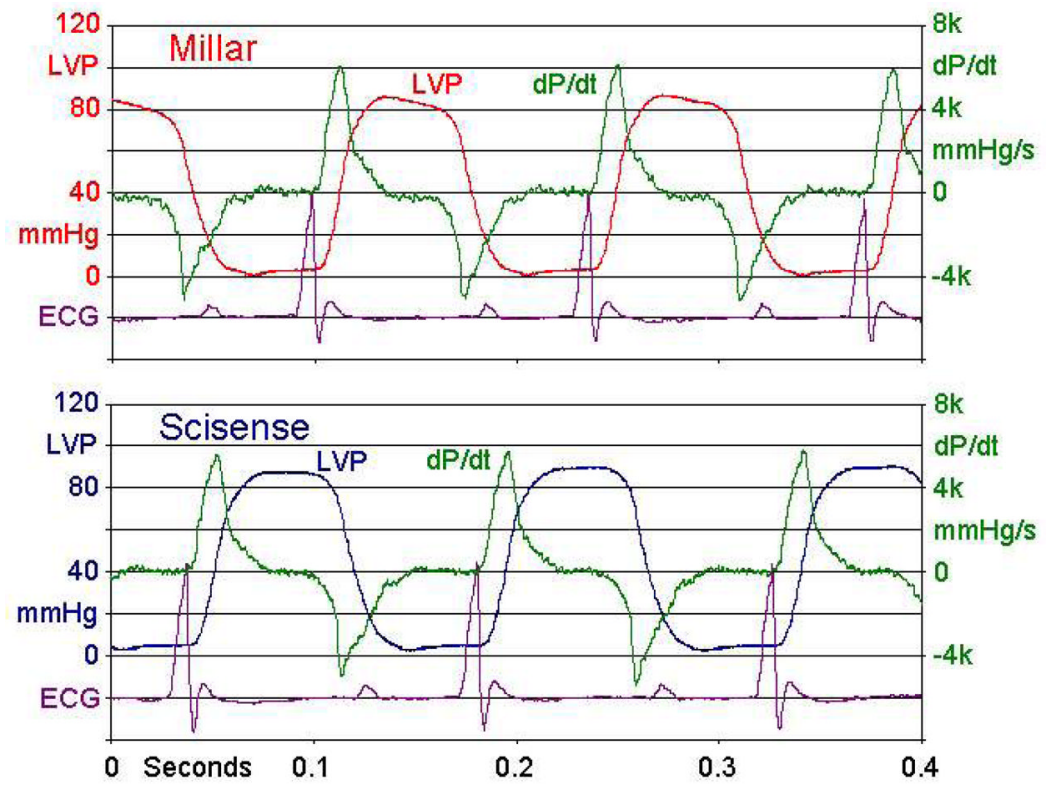

Figure 9.

Pressure and $\mathrm{dP} / \mathrm{dt}$ signals taken with Millar and Scisense catheters inserted sequentially into the LV of a mouse via the right carotid artery. 